SCIENTIFIC REPORT

\title{
Fluorescein and indocyanine green angiography findings in Behçet's disease
}

\author{
L S Atmaca, P A Sonmez
}

Br J Ophthalmol 2003;87:1466-1468

Aim: To evaluate and compare the fluorescein (FA) and indocyanine green angiographic (ICGA) findings, and to investigate the choroidal involvement in Behçet's disease.

Methods: FA and ICGA were performed on 112 eyes of 62 patients with Behçet's disease, between November 1993 and July 2002, using Topcon IMAGEnet Digital System. Patients were aged 16-50 years; 48 (77.4\%) were male and 14 $(22.6 \%)$ were female.

Results: FA showed dye leakage from retinal vessels in 57 (50.9\%) eyes, cystoid macular oedema in 18 eyes (16.1\%), optic disc oedema in four eyes (3.6\%), disc neovascularisation in three eyes $(2.7 \%)$, and retinal neovascularisation in two eyes (1.8\%). ICGA showed hyperfluorescent lesions in 40 eyes $(35.7 \%)$, hypofluorescent lesions in 17 eyes $(15.2 \%)$, hyperfluorescent and hypofluorescent lesions in 12 (10.7\%) eyes, ICG leakage from choroidal vessels in 11 eyes $(9.8 \%)$, irregular filling of choriocapillaris in five eyes $(4.5 \%)$, and choroidal filling defect in four eyes (3.6\%). Hyperfluorescent and hypofluorescent lesions which were seen in 53 out of 69 eyes $(76.8 \%)$ on ICGA, were not visible on FA. 55 eyes (49.1\%) showed hyperfluorescence on the disc on both FA and ICGA, whereas 36 (32.1\%) showed hyperfluorescence only on FA.

Conclusion: The hyperfluorescence and/or hypofluorescence, irregular filling of the choriocapillaris, choroidal filling defect, and ICG leakage from choroidal vessels seen only on ICGA may suggest choroidal involvement in Behçet's disease.

B ehçet's disease is named after Hulusi Behçet, a Turkish dermatologist, who in 1937 recognised recurrent oral and genital ulcers and iridocyclitis as a separate clinical entity. ${ }^{1}$ It is a chronic, multisystem disease with an unknown aetiology. Ocular involvement is reported to be $23-96 \%$ in different studies. ${ }^{23}$ Although the most common ocular symptom is anterior uveitis, obliterative and necrotising vasculitis is the characteristic histopathological hallmark of Behçet's disease. The retinal vasculature is often involved first either as periphlebitis or arteritis obliterans. The incidence of posterior segment involvement is reported to be $50-93 \% .^{45}$ The fundus findings of Behçet's disease are vitreal cellular infiltrates, venous and capillary dilatation, central or branch retinal vein occlusion, retinal oedema, macular oedema-especially cystoid type, optic disc hyperaemia and oedema. Macular hole, attenuated or ghost vessels, retinal and/or disc neovascularisations, and vitreous haemorrhage can also be seen. Tractional retinal detachment, retinal atrophy, papilloedema, and optic atrophy can be seen in the later stages of the disease.

Fluorescein angiography (FA) contributes greatly to the early diagnosis of the disease and is essential in the long term care of the patients. Fluorescein leakage from retinal vessels may be seen before there are obvious ophthalmoscopic signs of vasculitis. ${ }^{5}$ Dye leakage from the papillary and retinal capillaries, vascular wall staining, retinal vein occlusion, cystoid macular oedema, vascular remodelling as a result of capillary non-perfusion, and retinal and/or disc neovascularisation is well documented by FA.

In spite of these well known ophthalmoscopic and fluorescein angiographic findings for retinal involvement in Behçet's disease, the extent of choroidal involvement on ICGA is yet to be determined. Therefore, in this study, we would like to present our fluorescein and ICG angiographic findings for retinal and choroidal involvement in Behçet's disease.

\section{PATIENTS AND METHODS}

In all, 112 eyes of 62 patients who had previously been diagnosed with Behçet's disease were included in this study. All patients fulfilled the diagnostic criteria of the International Study Group for Behçet's disease. ${ }^{6}$ Consecutive fluorescein and ICG angiographies were performed on all patients, between November 1993 and July 2002, using Topcon IMAGEnet-H1024 Digital Imaging System. Of the patients, $48(77.4 \%)$ were male, and 14 $(22.6 \%)$ were female. Patients were aged between 1650 years (mean age 29.3).

Fluorescein angiograms were taken after a bolus injection of $5 \mathrm{ml}$ of $10 \%$ fluorescein via cubital vein. Early (up to 3 minutes), mid (9-13 minutes), and late (30-35 minutes) phase ICG angiograms followed FA, after an injection of $25 \mathrm{mg}$ ICG dye. All the angiograms were taken and evaluated by the same physician (LSA). Although many patients had several angiographies taken, only the first angiograms were taken into consideration.

\section{RESULTS}

Evaluation of the fluorescein angiograms showed dye leakage from retinal vessels in 57 (50.9\%) eyes, cystoid macular oedema in 18 eyes $(16.1 \%)$, chorioretinitis in nine eyes $(8 \%)$, optic disc oedema in four eyes (3.6\%), disc neovascularisation in three eyes $(2.7 \%)$, branch retinal vein occlusion in three eyes $(2.7 \%)$, retinal neovascularisation in two eyes $(1.8 \%)$, central retinal arterial occlusion in one eye $(0.9 \%)$, pigment epithelial detachment (PED) in one eye $(0.9 \%)$, and chorioretinal folds in one eye $(0.9 \%)$.

The evaluation of the ICG angiograms showed hyperfluorescent lesions in 40 eyes (35.7\%), hypofluorescent lesions in 17 eyes (15.2\%), hyperfluorescent and hypofluorescent lesions in $12(10.7 \%)$ eyes, hypofluorescence due to chorioretinitis scar in nine eyes $(8 \%)$, hypofluorescence due to PED in one eye $(0.9 \%)$, ICG leakage from choroidal vessels in 11 eyes $(9.8 \%)$ (Fig 1), irregular filling of choriocapillaris in five eyes $(4.5 \%)$ (Fig 2 ), and choroidal filling defect in four eyes (3.6\%) (Fig 3). 


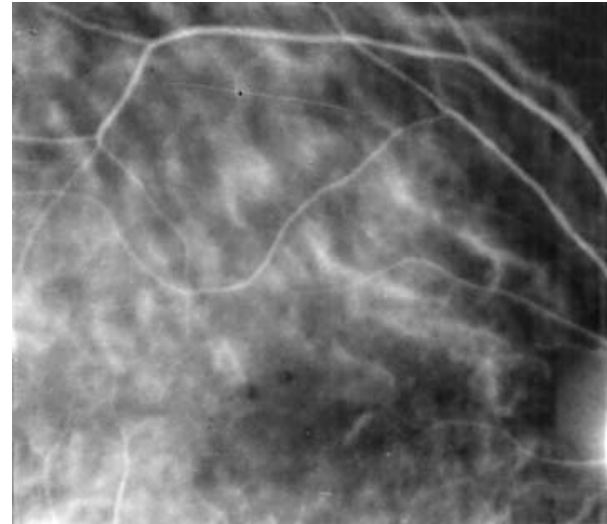

Figure 1 ICG leakage from choroidal vessels in early phase ICGA.

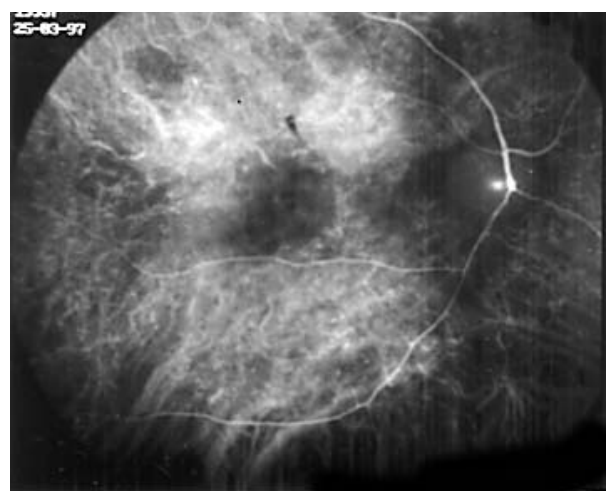

Figure 2 Irregular filling of the choriocapillaris in early phase ICGA.

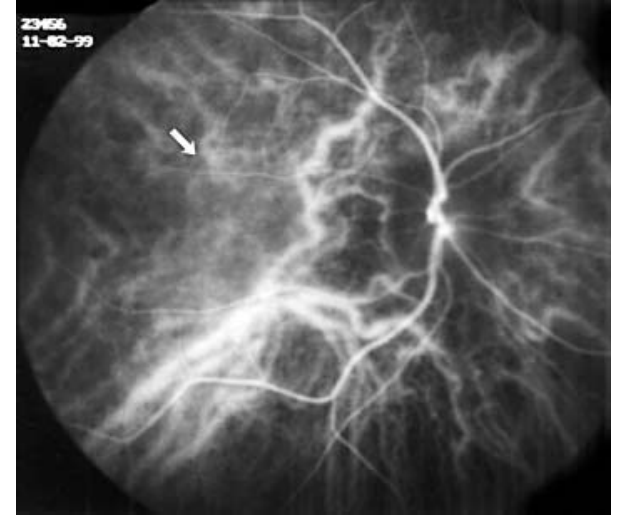

Figure 3 Choroidal filling defect in early phase ICGA (arrow).

Of the 112 eyes, 55 (49.1\%) showed hyperfluorescence on the disc both on FA and ICGA, whereas 36 (32.1\%) showed hyperfluorescence only on FA. Optic disc appeared normal both on FA and ICGA in 17 (15.2\%) eyes. The optic discs in the remaining four eyes $(3.6 \%)$ were atrophic.

Hyperfluorescent (Fig 4A, B), hypofluorescent (Fig 5A, B), hyperfluorescent and hypofluorescent lesions which were seen in 53 out of 69 eyes (76.8\%) on ICGA, were not visible on FA.

\section{DISCUSSION}

The retina is known to be the predominantly affected site of inflammation in posterior Behçet's disease. The fluorescein angiographic findings of Behçet's disease, which represent the retinal changes, have been well documented in the literature. Although the importance of choroidal circulation to support sensory retina with nourishment and oxygenation has long been recognised, there are only few studies in Behçet's disease documenting the indocyanine green angiographic
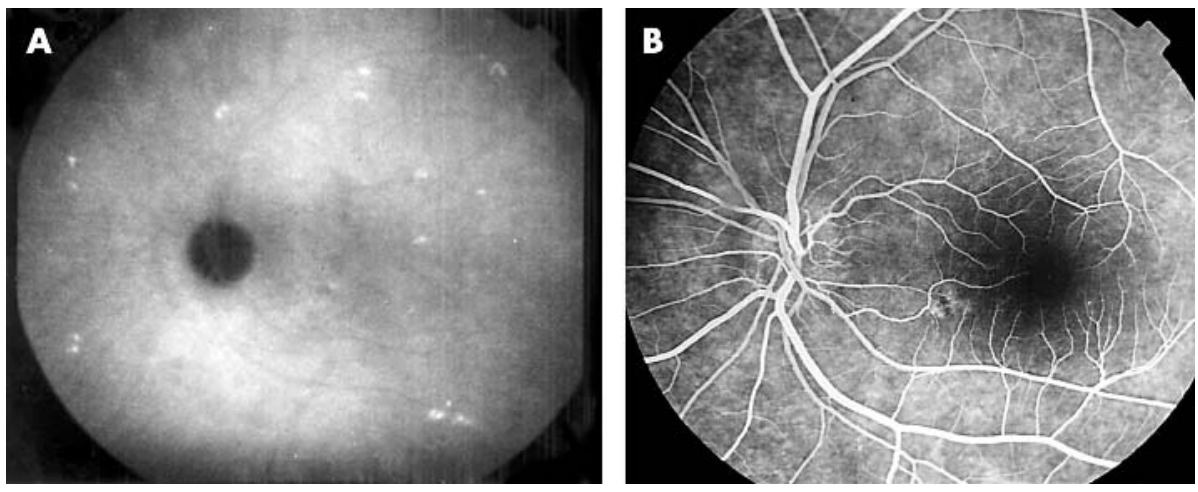

Figure 4 (A) Hyperfluorescent spots in the late phase of ICGA. (B) These spots cannot be seen on FA.
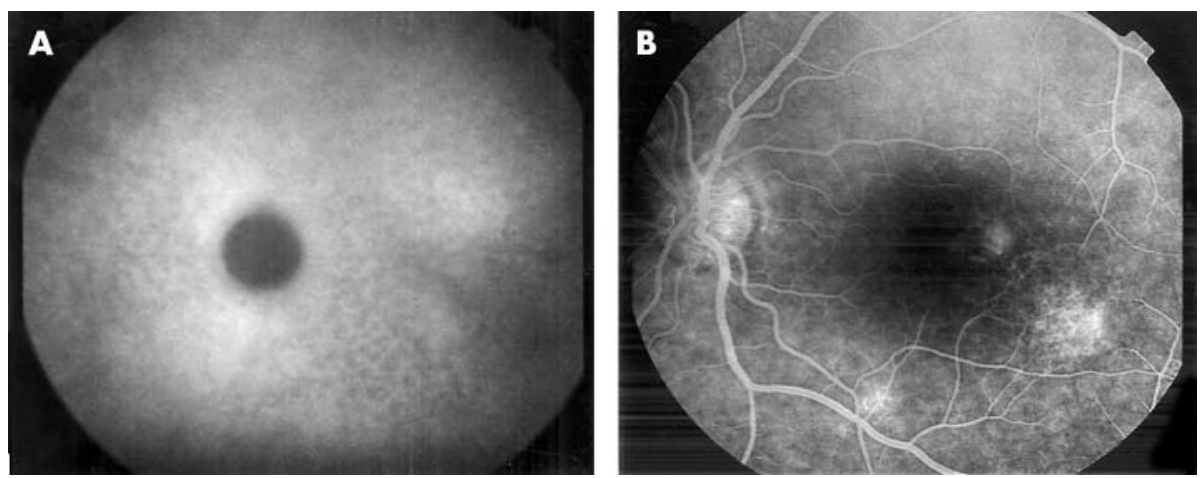

Figure 5 (A) Hypofluorescent spots in the late phase of ICGA. (B) These spots cannot be seen on FA. 
findings. ${ }^{78}$ ICGA allows choroidal vasculature to be observed better than FA, since ICG shows less leakage from the choriocapillaris and has longer wavelengths for emission and excitation to transcend the retinal pigment epithelium. ${ }^{7}$

Histopathological studies on eyes with Behçet's disease showed intensive retinal vasculitis and diffuse or focal infiltration of the choroid with inflammatory cells but no choroidal vasculitis. ${ }^{9}{ }^{10}$ However, one study reports immunoglobulin and complement deposition in choroidal veins. ${ }^{11}$

The ICG angiographic findings we have observed in this study were hyperfluorescent lesions (35.7\%), hypofluorescent lesions (15.2\%), hyperfluorescent and hypofluorescent lesions, (10.7\%), ICG leakage from choroidal vessels $(9.8 \%)$, irregular ICG filling of the choriocapillaris (4.5\%), and choroidal filling defects $(3.6 \%)$.

The hyperfluorescence and/or hypofluorescence seen both on FA and ICGA probably represent changes secondary to retinal inflammation. However, $76.8 \%$ of these hyperfluorescent and hypofluorescent lesions seen on ICGA were not evident on FA, which suggests that these may reflect primary choroidal involvement as well as ICG leakage from choroidal vessels, irregular ICG filling of the choriocapillaris, and choroidal filling defects. Choroidal hyperfluorescence on ICGA is either due to leakage, observed in any inflammatory disease involving choroid or to tissue staining, observed in granulomatous choroiditis. ${ }^{12}$ Matsuda et al ${ }^{13}$ show that leucocytes have an affinity for ICG dye and therefore suggested that the hyperfluorescent dots may be due to leucocyte accumulation.

Hypofluorescence on ICGA is seen as a result of blockage of the fluorescence, chorioretinal atrophy, non-perfusion of the choriocapillaris, or impaired choroidal ICG diffusion. ${ }^{12}$ In Behçet's disease, blockage of the fluorescence may be caused by oedema and fibrosis commonly seen in the choroid. Hypofluorescent lesions seen only on ICGA cannot be attributed to chorioretinal atrophy, since they would appear hyperfluorescent on FA. Choriocapillaris non-perfusion as a result of choroidal vascular obstruction may be another reason for hypofluorescence, although rarely shown histopathologically. Impaired choroidal ICG diffusion may be due to choroidal stromal scarring where hypofluorescence would be persistent; or to stromal inflammation where it would show resolution. Herbort et $a l^{12}$ reported that the hypofluorescent lesions seen at all phases of ICGA but not on FA, may represent chorioretinal stromal atrophy after choroidal inflammation with an intact, undamaged retinal pigment epithelium.

In Matsuo et al's study, involving 63 eyes of 33 patients with Behçet's disease, $66 \%$ of eyes showed hyperfluorescent lesions and 35\% showed hypofluorescent lesions on ICGA but not on FA. Twenty five per cent of eyes showed choroidal vascular wall staining, 5\% showed ICG leakage from choroidal vessels, and $17 \%$ showed irregular filling of the choriocapillaris. Eighty four per cent of eyes showed fluorescein leakage from retinal vessels. When comparing our study with that of Matsuo et al, one difference was the type of imaging system used. In our study, we used the conventional fundus camera linked to a digital system, while Matsuo et al used the confocal scanning laser ophthalmoscopy-which is not considered as useful as the digital system for patients with uveitis. ${ }^{8}$ Another difference in our study was the absence of choroidal vascular wall staining on ICGA.

Klaeger $e a^{8} l^{8}$ performed FA and ICGA on five patients with Behçet's disease at initial examination and after initiation of therapy. They included only patients with active disease to limit their study to primary inflammatory events. They noted fuzziness of choroidal vessels and choroidal hyperfluorescence due to leakage, indicating choroidal vasculitis in all their patients. They reported that in all four patients followed up by ICGA, fuzziness of choroidal vessels had responded to therapy. One patient had severe retinal involvement only in one eye, but choroidal involvement in both eyes. This finding may indicate that choroidal involvement occurs more frequently than we can detect but responds to therapy quicker and easier than retinal involvement. In their study, they did not observe any hypofluorescent spots. Therefore, they suggested that choroidal involvement in Behçet's disease may be limited to a pure vasculitis without stromal involvement. In our study, however, we found hypofluorescent lesions in 15.2\%, hyperfluorescent and hypofluorescent lesions in $10.7 \%$ of the eyes. Therefore, the findings of only five patients may not be sufficient to reach such a conclusion. They also observed disc hyperfluorescence in four of the five patients, indicating severe inflammation of the papillary vessels. In our study, $49.1 \%$ of eyes showed hyperfluorescence on the disc both on FA and ICGA, whereas $36.1 \%$ showed hyperfluorescence only on FA. Klaeger et al found delay in choriocapillaris perfusion in all cases, which was less pronounced after treatment.

Our findings such as hyperfluorescent and/or hypofluorescent lesions, irregular filling of choriocapillaris, choroidal filling defects, and leakage from choroidal vessels seen only on ICGA most likely represent choroidal involvement in Behçet's disease, which may indicate a more diffuse or severe disease. This study, along with the ones in the literature, indicates that although the retina is the predominantly affected site of inflammation in Behçet's disease, the choroid is also involved. However, these findings need to be strengthened by other histopathological and clinical studies. ICGA together with FA would provide useful information on ocular involvement in Behçet's disease.

\section{Authors' affiliations}

L S Atmaca, Ankara University, School of Medicine, Department of Ophthalmology, Gazi Mustafa Kemal Bulvari 23/1, Kizilay 06440, Ankara, Turkey

P A Sonmez, Gazi Mustafa Kemal Bulvari 23/1, Kizilay 06440, Ankara, Turkey

Correspondence to: Professor Leyla S Atmaca, Gazi Mustafa Kemal Bulvari 23/1, Kizilay 06440, Ankara, Turkey; leylaatmaca@tnnet.net.tr

Accepted for publication 17 March 2003

\section{REFERENCES}

1 Behçet H. Über rez idivierende, aphthose, durch ein virus verursachte Geschwure am Munde, am Auge und an Genitalien. Dermatol Wochenschr 1937; 105:1152-7.

2 Haim S, Barzilai D, Hazani E. Involvement of veins in Behçet's syndrome. Br J Dermatol 1971;84:238-41.

3 Mamo JG, Baghdassarian A. Behçet's disease: a report of 28 cases. Arch Ophthalmol 1964;71:4-14.

4 Mavioglu H. Behçet's recurrent disease. Analytical review of the literature. Missouri Med 1958;55:1209-22.

5 Atmaca LS. Fundus changes associated with Behçet's disease. Graefes Arch Clin Exp Ophthalmol 1989;227:340-4.

6 International Study Group for Behçet's Disease. Criteria for diagnosis of Behçet's disease. Lancet 1990;335:1078-80.

7 Matsuo T, Sato Y, Shiraga F, et al. Choroidal abnormalities in Behçet's disease observed by simultaneous indocyanine green and fluorescein angiography with scanning laser ophthalmoscopy. Ophthalmology 1999;106:295-300.

8 Klaeger A, Tran TV, Hiroz CA, et al. Indocyanine green angiography in Behçet's uveitis. Retina 2000;20:309-14.

9 Charteris DG, Champ C, Rosenthal AR, et al. Behçet's disease: activated T lymphocytes in retinal perivasculitis. Br J Ophthalmol 1992;76:499-501.

10 George RK, Chan CC, Whitcup SM, et al. Ocular immunopathology of Behçet's disease. Surv Ophthalmol 1997;42:157-62.

11 Mullaney J, Collum LMT. Ocular vasculitis in Behçet's disease. A pathological and immunohistochemical study. Int Ophthalmol 1985;7:183-91.

12 Herbort CP, LeHoang P, Guex-Croiser Y. Schematic interpretation of indocyanine green angiography in posterior uveitis using a standart angiographic protocol. Ophthalmology 1998;105:432-40.

13 Matsuda N, Ogura Y, Nishiwaki $H$, et al. Visualisation of leukocyte dynamics in the choroid with indocyanine green. Invest Ophthalmol Vis Sci 1996;37:2228-33. 